\title{
THIRD COMPONENT SEARCH AND ABUNDANCES OF THE VERY DUSTY SHORT-PERIOD BINARY BD $+20^{\circ} 307$
}

\author{
Francis C. Fekel ${ }^{1,6}$, Maria J. Cordero ${ }^{2}$, Raphael Galicher ${ }^{3,7}$, B. Zuckerman ${ }^{4}$, \\ CARl Melis ${ }^{4,8}$, and Alycia J. Weinberger ${ }^{5}$ \\ ${ }^{1}$ Center of Excellence in Information Systems, Tennessee State University, 3500 John A. Merritt Boulevard, Box 9501, \\ Nashville, TN 37209, USA; fekel@evans.tsuniv.edu \\ ${ }^{2}$ Department of Astronomy, Indiana University, Bloomington, IN 47405, USA; majocord@indiana.edu \\ ${ }^{3}$ National Research Council Canada, Herzberg Institute of Astrophysics, 5071 West Saanich Road, Victoria, BC V9E 2E7, Canada; raphael.galicher@ nrc-cnrc.gc.ca \\ ${ }^{4}$ Department of Physics and Astronomy, University of California, Los Angeles, CA 90095-1547, USA; ben@ astro.ucla.edu, cmelis@ucsd.edu \\ ${ }^{5}$ Department of Terrestrial Magnetism, Carnegie Institution for Science, 5241 Broad Branch Road NW, Washington, DC 20015, USA; weinberger@ dtm.ciw.edu \\ Received 2011 November 23; accepted 2012 January 26; published 2012 March 16
}

\begin{abstract}
We have obtained near-infrared adaptive optics imaging and collected additional radial velocity observations to search for a third component in the extremely dusty short-period binary system $\mathrm{BD}+20^{\circ} 307$. Our image shows no evidence for a third component at separations greater than $19 \mathrm{AU}$. Our four seasons of radial velocities have a constant center-of-mass velocity and are consistent with the systemic velocities determined at two earlier epochs. Thus, the radial velocities also provide no support for a third component. Unfortunately, the separation domains covered by our imaging and radial velocity results do not overlap. Thus, we examined the parameters for possible orbits of a third component that could have been missed by our current observations. With our velocities we determined improved circular orbital elements for the 3.4 day double-lined binary. We also performed a spectroscopic abundance analysis of the short-period binary components and conclude that the stars are a mid- and a late-F dwarf. We find that the iron abundances of both components, $[\mathrm{Fe} / \mathrm{H}]=0.15$, are somewhat greater than the solar value and comparable to that of stars in the Hyades. Despite the similarity of the binary components, the lithium abundances of the two stars are very unequal. The primary has $\log \epsilon(\mathrm{Li})=2.72$, while in the secondary $\log \epsilon(\mathrm{Li}) \leqslant 1$.46, which corresponds to a difference of at least a factor of 18 . The very disparate lithium abundances in very similar stars make it impossible to ascribe a single age to them. While the system is likely at least $1 \mathrm{Gyr}$ old, it may well be as old as the Sun.
\end{abstract}

Key words: binaries: spectroscopic - stars: abundances - stars: fundamental parameters - stars: individual (BD $\left.+20^{\circ} 307\right)$

Online-only material: machine-readable table

\section{INTRODUCTION}

Whitelock et al. (1991) and Stencel \& Backman (1991) first identified BD $+20^{\circ} 307=$ HIP $8920\left(\alpha=01^{\mathrm{h}} 54^{\mathrm{m}} 50\right.$ s $34, \delta=$ $+21^{\circ} 18^{\prime} 22^{\prime \prime} .5$ [2000.0]) as a solar-type star with a very large near-infrared excess. Song et al. (2005) obtained infrared spectra in the $8-13 \mu \mathrm{m}$ region that showed its strong silicate emission features. They concluded that the extremely large amount of warm dust around $\mathrm{BD}+20^{\circ} 307$ likely resulted from the recent collisions of asteroids or planetesimals and suggested that the system was rather young with an age of $\sim 300 \mathrm{Myr}$.

Believing $\mathrm{BD}+20^{\circ} 307$ to be a single star, Zuckerman et al. (2008) began obtaining X-ray, photometric, and spectroscopic observations to improve the age estimate of the system and thus constrain the epoch of planet formation. However, Weinberger (2008) discovered that the star is a 3.4 day, double-lined spectroscopic binary. Unfortunately, the fact that $\mathrm{BD}+20^{\circ} 307$ is a short-period binary invalidates the use of $\mathrm{X}$-ray and rotation period measurements to estimate its age.

Zuckerman et al. (2008) concluded from the system's $B-V$ color that the components of $\mathrm{BD}+20^{\circ} 307$ are somewhat

\footnotetext{
6 Visiting Astronomer, Kitt Peak National Observatory, National Optical Astronomy Observatory, operated by the Association of Universities for Research in Astronomy, Inc., under cooperative agreement with the National Science Foundation.

7 Department de Physique, Université de Montréal, C.P. 6128 Succ. Centre-ville, Montréal, QC H3C 3J7, Canada.

8 Current address: Center for Astrophysics and Space Sciences, University of California, San Diego, CA 92093-0424, USA.
}

metal-poor relative to the Sun and that the lithium abundance difference between the two components indicates an age of one to several Gyr. Thus, they argued that the extensive amount of warm dust in the $\mathrm{BD}+20^{\circ} 307$ system does not result from a very young star beginning the process of making planets, but rather comes from the recent collision of planets in a relatively mature main-sequence system. The disruption of the orbits of such planetary-sized objects might be caused by a third body. Indeed, according to the survey of Tokovinin et al. (2006), short-period binaries are very likely to be systems of higher multiplicity.

In this follow-up paper we have used adaptive optics (AO) imaging and continued radial velocity monitoring to search for a third star in the system. The new spectroscopic observations have enabled us to improve the 3.4 day binary orbit. In addition, we have performed an abundance analysis of an echelle spectrogram to examine in a more rigorous way the conclusion that $\mathrm{BD}+20^{\circ} 307$ has an iron underabundance. From that spectrum, we have determined lithium abundances for the two components and further consider the lithium difference discussed by Weinberger (2008) and Zuckerman et al. (2008).

\section{OBSERVATIONS}

\subsection{Adaptive Optics Imaging}

On 2008 July 11, we obtained high angular resolution images of $\mathrm{BD}+20^{\circ} 307$ with the Keck II telescope of the Keck Observatory using its AO system and the NIRC2 near-infrared narrow-field camera (McLean \& Sprayberry 2003) in the 


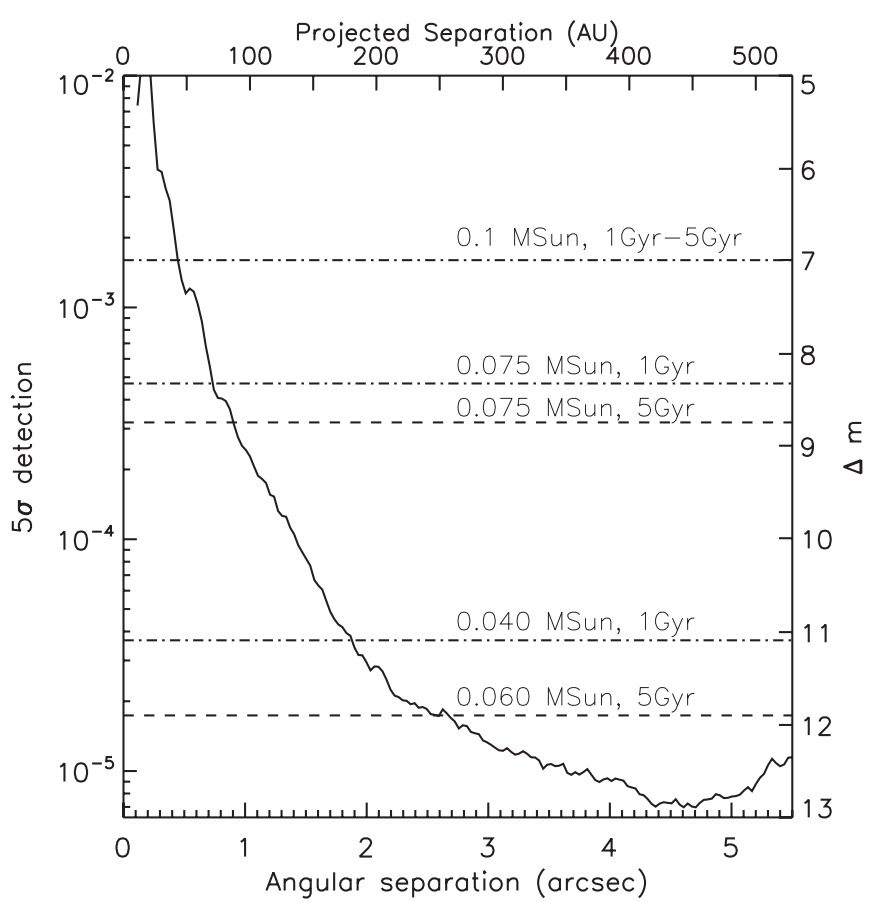

Figure 1. The $5 \sigma$ contrast profile detection limit as a function of angular separation for our $K p$-bandpass Keck II NIRC2 AO data. Dot-dashed and dashed lines indicate the magnitude difference level between $\mathrm{BD}+20^{\circ} 307$ and a putative companion for various mass and age combinations.

$K p$-bandpass, which is centered at $2.124 \mu \mathrm{m}$. No coronagraph was used for these observations. We have obtained one $1024 \times 1024(\sim 10 \times 10$ arcsec field $)$ saturated image $(2$ co-adds of $30 \mathrm{~s})$ and three $64 \times 120$ unsaturated images $(50$ co-adds of $5 \mathrm{~ms}$ ).

Data reduction of the $\mathrm{AO}$ images was performed as follows: we first subtracted a dark image, then divided by a flat field, and next masked the bad/hot pixels. We then removed known NIRC2 narrow-field camera distortions using the Yelda et al. (2010) solution. The images were rotated by 0.252 clockwise to put north up, and the plate scale was selected to be $9.952 \pm$ 0.002 mas pixel $^{-1}$ to be consistent with the distortion correction of Yelda et al. (2010). After these steps, we registered the unsaturated images at the detector center and used their median and the integrated point-spread function core intensity to fluxnormalize the saturated images. The contrast curve as a function of angular separation was obtained by calculating the standard deviation in a small $\lambda / D$ width annulus of increasing radius. Figure 1 presents the $5 \sigma$ contrast profile, which is discussed in Section 3.1 .

\subsection{Optical Spectroscopy}

From 2008 January to 2011 January, we collected 64 usable observations with the Tennessee State University $2 \mathrm{~m}$ automatic spectroscopic telescope (AST), fiber-fed echelle spectrograph, and a $2048 \times 4096$ SITe ST-002A CCD. The echelle spectrograms have 21 orders, covering the wavelength range 4920-7100 A. Work done on the telescope-spectrograph system in early 2010 resulted in observations with improved signal-tonoise ratios after 2010 January. Before that date the spectra had a resolution of $0.17 \AA$, while afterward the resolution was decreased to $0.3 \AA$, enabling signal-to-noise ratios of $\sim 40$ at $6000 \AA$ to be obtained in these more recent observations. Eaton $\&$ Williamson $(2004,2007)$ have given an extensive description of the telescope and spectrograph, situated at Fairborn Observatory near Washington Camp in the Patagonia Mountains of southeastern Arizona, and its operation.

During 2008 and 2009, we acquired five red wavelength observations of BD $+20^{\circ} 307$ with the Kitt Peak National Observatory (KPNO) coudé feed telescope, coudé spectrograph, and CCD detectors. In 2008 September, we obtained two spectrograms with the KPNO T1KA CCD. Those observations are centered at $6400 \AA$, cover a wavelength range of $172 \AA$, and have a resolution of $0.34 \AA$. One year later, we acquired three additional spectra with the KPNO TI5 CCD. Those spectrograms are centered at $6430 \AA$, cover a wavelength range of $84 \AA$, and have a resolution of $0.21 \AA$. The spectra have signalto-noise ratios of about 100 .

In 2008, we obtained three echelle spectra at Lick Observatory with the Shane $3 \mathrm{~m}$ telescope and Hamilton echelle spectrograph (Vogt 1987). The spectra cover the wavelength range 3810-7900 $\AA$ at a resolution of typically $0.17 \AA$ in the red region of the spectrum (resolving power 38,000). One of the spectra was acquired specifically for an abundance analysis and was not measured for radial velocities.

Zuckerman et al. (2008) discussed the reduction and velocity measurement of the Fairborn Observatory AST echelle spectra. The KPNO radial velocities were determined with the IRAF cross-correlation program FXCOR (Fitzpatrick 1993). Two International Astronomical Union radial velocity standard stars, $\beta$ Vir and 10 Tau, having adopted velocities of 4.4 and $27.9 \mathrm{~km} \mathrm{~s}^{-1}$ (Scarfe et al. 1990), respectively, were used as crosscorrelation reference stars. Reduction of the Hamilton echelle spectra with IRAF tasks has been discussed in detail in Lick Technical Report No. 74. ${ }^{9}$ Briefly, the stellar spectra have been bias subtracted, flat-fielded, extracted, and finally wavelength calibrated with Th-Ar emission line comparison spectra. Radial velocities of the Lick Observatory spectra were determined by cross-correlation with HD 9224 used as the standard star. From Nidever et al. (2002), we adopted a velocity of $14.9 \mathrm{~km} \mathrm{~s}^{-1}$ for the standard. Table 1 lists the heliocentric Julian dates of the 71 spectra that were measured for radial velocities and gives the velocities of the primary and secondary components.

\section{SEARCH FOR A THIRD COMPONENT}

\subsection{Adaptive Optics Imaging}

As noted previously, Figure 1 shows the $5 \sigma$ contrast profile for $\mathrm{BD}+20^{\circ} 307$. Unfortunately, our data are saturated at angular separations of less than $0^{\prime \prime} 2$. For a given separation that profile shows the minimum brightness of an object that could be seen relative to the primary. The reanalyzed Hipparcos parallax (van Leeuwen 2007) gives a distance of $96 \pm 12$ pc from the Sun, and so the angular separation of 0 '. 2 corresponds to a linear projected separation of $19 \mathrm{AU}$ where it is possible to search for a third component. At a projected separation of $\sim 45 \mathrm{AU}$ a late-M dwarf would have been found, while at larger projected separations of 75-95 AU (for 1 and 5 Gyr, dusty models; Chabrier et al. 2000), we would have detected a star of any mass, right down to the stellar/brown dwarf boundary. At $\sim 200$ AU or greater, we can detect a brown dwarf that is less than 40-60 Jupiter masses (again for ages of 1 and $5 \mathrm{Gyr}$ ). There is no evidence for any of those companions in our image.

\footnotetext{
9 http://astronomy.nmsu.edu/cwc/Software/irafman/manual.html
} 
Table 1

Radial Velocities and Orbital Residuals

\begin{tabular}{|c|c|c|c|c|c|c|c|c|}
\hline $\begin{array}{l}\text { Hel. Julian Date } \\
\text { (HJD }-2,400,000)\end{array}$ & Phase & $\begin{array}{c}\mathrm{RV}_{1} \\
\left(\mathrm{~km} \mathrm{~s}^{-1}\right)\end{array}$ & $\begin{array}{l}(O-C)_{1} \\
\left(\mathrm{~km} \mathrm{~s}^{-1}\right)\end{array}$ & Weight $_{1}$ & $\begin{array}{c}\mathrm{RV}_{2} \\
\left(\mathrm{~km} \mathrm{~s}^{-1}\right)\end{array}$ & $\begin{array}{l}(O-C)_{2} \\
\left(\mathrm{~km} \mathrm{~s}^{-1}\right)\end{array}$ & Weight $_{2}$ & Source $^{\mathrm{a}}$ \\
\hline 54495.585 & 0.848 & 10.8 & -1.9 & 0.2 & -37.1 & 2.1 & 0.2 & Fair \\
\hline 54497.586 & 0.433 & -51.4 & 0.7 & 0.2 & 29.8 & -0.2 & 0.2 & Fair \\
\hline 54499.586 & 0.018 & 30.6 & -0.2 & 0.2 & -58.7 & -0.1 & 0.2 & Fair \\
\hline 54499.690 & 0.048 & 28.4 & -0.7 & 0.2 & -57.2 & -0.5 & 0.2 & Fair \\
\hline 54502.690 & 0.925 & 26.1 & -0.3 & 0.2 & -52.6 & 1.2 & 0.2 & Fair \\
\hline 54506.630 & 0.077 & 26.2 & 0.1 & 0.2 & -54.3 & -0.8 & 0.2 & Fair \\
\hline 54506.679 & 0.092 & 24.9 & 0.8 & 0.2 & -52.2 & -0.9 & 0.2 & Fair \\
\hline 54507.590 & 0.358 & -38.2 & 1.5 & 0.2 & 14.7 & -2.1 & 0.2 & Fair \\
\hline 54508.590 & 0.651 & -38.0 & -0.1 & 0.2 & 16.6 & 1.8 & 0.2 & Fair \\
\hline 54514.593 & 0.406 & -47.7 & 0.8 & 0.2 & 25.3 & -0.8 & 0.2 & Fair \\
\hline 54516.594 & 0.991 & 30.1 & -0.9 & 0.2 & -59.4 & -0.6 & 0.2 & Fair \\
\hline 54516.658 & 0.010 & 30.7 & -0.3 & 0.2 & -59.6 & -0.8 & 0.2 & Fair \\
\hline 54523.597 & 0.038 & 29.9 & 0.1 & 0.2 & -58.1 & -0.6 & 0.2 & Fair \\
\hline 54523.648 & 0.053 & 29.8 & 1.1 & 0.2 & -55.8 & 0.5 & 0.2 & Fair \\
\hline 54524.598 & 0.331 & -31.6 & 2.0 & 0.2 & 8.8 & -1.4 & 0.2 & Fair \\
\hline 54532.602 & 0.671 & -34.2 & -1.2 & 0.2 & 10.7 & 1.1 & 0.2 & Fair \\
\hline 54533.604 & 0.964 & 29.9 & -0.1 & 0.2 & -56.0 & 1.7 & 0.2 & Fair \\
\hline 54535.604 & 0.549 & -54.9 & -1.0 & 0.2 & 31.6 & -0.2 & 0.2 & Fair \\
\hline 54540.602 & 0.011 & 29.9 & -1.1 & 0.2 & -60.0 & -1.3 & 0.2 & Fair \\
\hline 54620.948 & 0.503 & -56.4 & -0.5 & 0.2 & 34.8 & 0.8 & 0.2 & Fair \\
\hline 54634.922 & 0.589 & -50.3 & -1.0 & 0.2 & 26.3 & -0.7 & 0.2 & Fair \\
\hline 54644.986 & 0.531 & -54.5 & 0.6 & 0.2 & 33.7 & 0.6 & 0.2 & Lick \\
\hline 54645.001 & 0.536 & -55.0 & -0.2 & 0.2 & 32.6 & -0.3 & 0.2 & Lick \\
\hline 54677.815 & 0.130 & 20.2 & 2.9 & 0.2 & -45.6 & -1.5 & 0.2 & Fair \\
\hline 54697.843 & 0.986 & 30.1 & -0.8 & 0.2 & -58.7 & 0.0 & 0.2 & Fair \\
\hline 54731.847 & 0.929 & 25.2 & -1.6 & 0.2 & -53.2 & 1.1 & 0.2 & KPNO \\
\hline 54733.846 & 0.513 & -56.1 & -0.3 & 0.2 & 33.3 & -0.6 & 0.2 & KPNO \\
\hline 54748.009 & 0.654 & -37.3 & -0.3 & 0.2 & 17.0 & 3.2 & 0.2 & Fair \\
\hline 54752.999 & 0.113 & 20.0 & -0.5 & 0.2 & -47.9 & -0.4 & 0.2 & Fair \\
\hline 54844.586 & 0.893 & 21.9 & 0.3 & 0.2 & -47.6 & 1.1 & 0.2 & Fair \\
\hline 54877.617 & 0.551 & -55.3 & -1.6 & 0.2 & 31.1 & -0.6 & 0.2 & Fair \\
\hline 55063.895 & 0.017 & 29.9 & -1.0 & 1.0 & -58.6 & 0.0 & 0.6 & Fair \\
\hline 55082.902 & 0.574 & -51.9 & -0.6 & 1.0 & 29.3 & 0.2 & 0.6 & Fair \\
\hline 55094.875 & 0.075 & 26.3 & -0.1 & 1.0 & -54.3 & -0.5 & 0.6 & KPNO \\
\hline 55095.952 & 0.390 & -45.2 & 0.7 & 1.0 & 23.1 & -0.2 & 0.6 & KPNO \\
\hline 55096.789 & 0.634 & -41.6 & -0.3 & 1.0 & 18.7 & 0.3 & 0.6 & KPNO \\
\hline 55126.604 & 0.352 & -38.1 & 0.3 & 1.0 & 15.1 & -0.3 & 0.6 & Fair \\
\hline 55154.595 & 0.536 & -55.2 & -0.4 & 1.0 & 32.7 & -0.1 & 0.6 & Fair \\
\hline 55178.585 & 0.551 & -54.2 & -0.5 & 1.0 & 30.6 & -1.1 & 0.6 & Fair \\
\hline 55243.614 & 0.565 & -52.6 & -0.2 & 1.0 & 30.6 & 0.3 & 0.6 & Fair \\
\hline 55244.621 & 0.859 & 14.9 & -0.2 & 1.0 & -42.0 & -0.2 & 0.6 & Fair \\
\hline 55245.624 & 0.152 & 12.6 & 0.0 & 1.0 & -39.3 & -0.2 & 0.6 & Fair \\
\hline 55246.638 & 0.449 & -53.6 & 0.1 & 1.0 & 32.3 & 0.6 & 0.6 & Fair \\
\hline 55270.611 & 0.458 & -54.6 & -0.2 & 1.0 & 31.9 & -0.5 & 0.6 & Fair \\
\hline 55364.941 & 0.040 & 29.9 & 0.1 & 1.0 & -57.2 & 0.2 & 0.6 & Fair \\
\hline 55365.941 & 0.332 & -33.9 & -0.1 & 1.0 & 10.6 & 0.1 & 0.6 & Fair \\
\hline 55366.941 & 0.624 & -42.8 & 0.5 & 1.0 & 21.1 & 0.5 & 0.6 & Fair \\
\hline 55368.949 & 0.211 & -2.2 & -0.2 & 1.0 & -23.5 & 0.0 & 0.6 & Fair \\
\hline 55374.887 & 0.948 & 29.2 & 0.4 & 1.0 & -56.9 & -0.5 & 0.6 & Fair \\
\hline 55376.887 & 0.532 & -55.1 & -0.1 & 1.0 & 32.8 & -0.3 & 0.6 & Fair \\
\hline 55377.882 & 0.823 & 7.6 & 0.7 & 1.0 & -33.1 & 0.0 & 0.6 & Fair \\
\hline 55384.930 & 0.884 & 20.3 & 0.2 & 1.0 & -47.6 & -0.5 & 0.6 & Fair \\
\hline 55385.953 & 0.183 & 5.4 & 0.1 & 1.0 & -31.6 & -0.3 & 0.6 & Fair \\
\hline 55412.892 & 0.060 & 28.2 & 0.2 & 1.0 & -56.0 & -0.4 & 0.6 & Fair \\
\hline 55432.923 & 0.917 & 25.7 & 0.4 & 1.0 & -52.7 & -0.1 & 0.6 & Fair \\
\hline 55442.913 & 0.838 & 10.9 & 0.5 & 1.0 & -36.9 & -0.1 & 0.6 & Fair \\
\hline 55443.931 & 0.135 & 16.6 & 0.3 & 1.0 & -42.5 & 0.5 & 0.6 & Fair \\
\hline 55444.912 & 0.422 & -50.6 & 0.2 & 1.0 & 29.4 & 0.8 & 0.6 & Fair \\
\hline 55450.910 & 0.176 & 7.0 & -0.1 & 1.0 & -33.0 & 0.2 & 0.6 & Fair \\
\hline 55453.914 & 0.054 & 28.0 & -0.6 & 1.0 & -56.0 & 0.2 & 0.6 & Fair \\
\hline 55454.882 & 0.337 & -35.5 & -0.4 & 1.0 & 12.5 & 0.7 & 0.6 & Fair \\
\hline 55455.881 & 0.630 & -41.8 & 0.5 & 1.0 & 19.7 & 0.2 & 0.6 & Fair \\
\hline 55469.024 & 0.472 & -55.3 & 0.0 & 1.0 & 33.1 & -0.2 & 0.6 & Fair \\
\hline 55477.858 & 0.055 & 28.4 & -0.1 & 1.0 & -55.5 & 0.6 & 0.6 & Fair \\
\hline 55482.906 & 0.531 & -54.8 & 0.3 & 1.0 & 32.6 & -0.5 & 0.6 & Fair \\
\hline
\end{tabular}


Table 1

(Continued)

\begin{tabular}{|c|c|c|c|c|c|c|c|c|}
\hline $\begin{array}{l}\text { Hel. Julian Date } \\
\text { (HJD - 2,400,000) }\end{array}$ & Phase & $\begin{array}{c}\mathrm{RV}_{1} \\
\left(\mathrm{~km} \mathrm{~s}^{-1}\right)\end{array}$ & $\begin{array}{l}(O-C)_{1} \\
\left(\mathrm{~km} \mathrm{~s}^{-1}\right)\end{array}$ & Weight $_{1}$ & $\begin{array}{c}\mathrm{RV}_{2} \\
\left(\mathrm{~km} \mathrm{~s}^{-1}\right)\end{array}$ & $\begin{array}{l}(O-C)_{2} \\
\left(\mathrm{~km} \mathrm{~s}^{-1}\right)\end{array}$ & Weight $_{2}$ & Source $^{\mathrm{a}}$ \\
\hline 55497.853 & 0.902 & 23.5 & 0.4 & 1.0 & -50.4 & -0.1 & 0.6 & Fair \\
\hline 55508.764 & 0.092 & 23.5 & -0.5 & 1.0 & -50.7 & 0.6 & 0.6 & Fair \\
\hline 55518.928 & 0.064 & 27.6 & 0.0 & 1.0 & -54.8 & 0.3 & 0.6 & Fair \\
\hline 55556.656 & 0.095 & 23.3 & -0.2 & 1.0 & -49.7 & 1.1 & 0.6 & Fair \\
\hline 55570.642 & 0.185 & 4.0 & -1.0 & 1.0 & -30.8 & 0.2 & 0.6 & Fair \\
\hline 55584.673 & 0.287 & -22.6 & -0.1 & 1.0 & -0.8 & 0.9 & 0.6 & Fair \\
\hline
\end{tabular}

Note. ${ }^{a}$ Fair: Fairborn Observatory; Lick: Lick Observatory; KPNO: Kitt Peak National Observatory.

Table 2

Center-of-mass Velocities

\begin{tabular}{lcccc}
\hline \hline $\begin{array}{l}\text { Data } \\
\text { Set }\end{array}$ & $\begin{array}{c}\text { Number of } \\
\text { Observations }\end{array}$ & $\begin{array}{c}\text { JD Range } \\
(\mathrm{JD}-2,400,000)\end{array}$ & $\begin{array}{c}\gamma \text { Velocity } \\
\left(\mathrm{km} \mathrm{s}^{-1}\right)\end{array}$ & Reference \\
\hline 1 & 1 & 53241 & $-11.0 \pm 1.0$ & Song et al. (2005) \\
2 & 3 & $54397-54399$ & $-12.3 \pm 0.2$ & This paper \\
3 & 19 & $54495-54540$ & $-12.43 \pm 0.11$ & This paper \\
4 & 12 & $54620-54877$ & $-12.46 \pm 0.23$ & This paper \\
5 & 13 & $55063-55270$ & $-12.57 \pm 0.08$ & This paper \\
6 & 27 & $55364-55584$ & $-12.29 \pm 0.05$ & This paper \\
\hline
\end{tabular}

\subsection{Radial Velocity}

We also searched for evidence of a third component by examining all our available radial velocities, which cover a longer time span and are much more extensive in number than those discussed by Zuckerman et al. (2008). We now have acquired four seasons of velocities, obtained primarily from Fairborn Observatory, but also including several velocities from KPNO and Lick Observatory. Using the orbital analysis programs described by Zuckerman et al. (2008) and the weights that they assigned to the component velocities, we initially obtained four double-lined circular orbit solutions, one for each season. The center-of-mass velocities of those orbits are very similar. However, because of the $2 \mathrm{~m}$ telescope system throughput improvements, which occurred in our third observing season, the Fairborn velocities that were obtained during the third and fourth seasons are more precise. So, we combined the data from our first and second seasons and computed two circular orbit solutions, one for the primary and the other for the secondary. We similarly combined the data from the third and fourth seasons and obtained two more orbital solutions. The variances of those solutions resulted in weights for the primary velocities of 0.2 for the first two seasons and 1.0 for the last two seasons. Similarly, for the secondary the weights are 0.2 and 0.6 , respectively. The appropriate weight for each individual velocity is listed in Table 1 . With the weighted velocities, we once again obtained double-lined circular orbit solutions for each of the four observing seasons. Our results are summarized in Table 2, which lists the number of observations in each of the four seasons, the Julian date range, and the centerof-mass velocity plus its uncertainty.

In addition to our velocities, there are two other sets that were obtained at earlier epochs. The first consists of a lone single-lined velocity measured by Song et al. (2005). The second set, only slightly more extensive, contains velocities from the three spectra of Weinberger (2008). Those six radial velocities, obtained nearly 100 days before the start of our own observations, result in a center-of-mass velocity of $-8.4 \mathrm{~km} \mathrm{~s}^{-1}$, a value that is $4 \mathrm{~km} \mathrm{~s}^{-1}$ more positive than our average systemic
Table 3

MIKE Remeasured Radial Velocities

\begin{tabular}{lrrr}
\hline \hline Component & \multicolumn{3}{c}{ HJD -2,400,000 } \\
\cline { 2 - 4 } & 54397.6969 & 54398.7041 & 54399.6810 \\
\hline Primary RV $\left(\mathrm{km} \mathrm{s}^{-1}\right)$ & $-3.6 \pm 0.8$ & $-55.4 \pm 0.5$ & $-0.1 \pm 0.5$ \\
Secondary RV $\left(\mathrm{km} \mathrm{s}^{-1}\right)$ & $-21.3 \pm 0.8$ & $34.0 \pm 0.5$ & $-25.7 \pm 0.5$ \\
\hline
\end{tabular}

velocity. Taken at face value, the large velocity shift over such a short period of time argues for a third component in an orbit with an extremely high eccentricity of 0.9 or greater.

The alternative possibility is that the velocity shift is spurious. Observatory zero-point differences often have been found to be in the 1-2 $\mathrm{km} \mathrm{s}^{-1}$ range (e.g., Harper 1932), while more modern results usually agree to better than $1 \mathrm{~km} \mathrm{~s}^{-1}$ (e.g., Scarfe et al. 1990; Nordström et al. 1994). Such differences may arise from a variety of factors, including the spectrograph system and temperature and mechanical flexure effects, as well as the reduction method.

In the velocity reduction of Weinberger (2008) the three spectra, obtained with the Magellan Inamori Kyocera Echelle (MIKE) spectrograph on the Magellan II telescope, were wavelength calibrated with the use of a ThAr spectrum that had been taken far in time from the stellar observations, and so velocity offsets resulting from internal instrumental drifts were not removed. To solve this problem, in our new reduction we have cross-correlated the spectral region of the oxygen $B$ band, 6884-6909 A, in all our spectra and removed the velocity offset measured there from all other echelle orders. In addition, instead of using a theoretical stellar atmosphere model as the radial velocity template, we have used another star, HD 197076, which was observed on 2007 October 23 and thus earlier in the same night as the first of the three $\mathrm{BD}+20^{\circ} 307$ MIKE observations. We used this G5V star as a radial velocity standard, adopting a velocity of $-35.4 \mathrm{~km} \mathrm{~s}^{-1}$ (with an uncertainty $<0.1 \mathrm{~km} \mathrm{~s}^{-1}$ ) from Nidever et al. (2002). We cross-correlated the BD $+20^{\circ} 307$ data on each of three nights (2007 October 24-26) against this reference star, using IRAF's FXCOR task to deblend the binary components of the correlation peaks. Table 3 is a corrected version of Table 2 from Weinberger (2008) and lists our remeasured velocities. The new reduction produces an orbit with a systemic velocity of $-12.3 \mathrm{~km} \mathrm{~s}^{-1}$, which is nearly $4 \mathrm{~km} \mathrm{~s}^{-1}$ more negative than the initially reported value. That newly computed systemic velocity plus the velocity of Song et al. (2005) are both listed in Table 2.

Figure 2 is a plot of the six center-of-mass velocities versus their Julian date mid-epochs. The systemic velocities for our four observing seasons have uncertainties of $0.2 \mathrm{~km} \mathrm{~s}^{-1}$ or less and result in a weighted average of $-12.38 \pm 0.03 \mathrm{~km} \mathrm{~s}^{-1}$. They show no evidence of orbital motion about a third body. 
Table 4

Short-period Orbital Elements and Related Parameters

\begin{tabular}{lcr}
\hline \hline Parameter & Zuckerman et al. (2008) & This Study \\
\hline$P($ days $)$ & $3.42015 \pm 0.00067$ & $3.420082 \pm 0.000012$ \\
$T_{0}^{\mathrm{a}}(\mathrm{HJD})$ & $2454506.3855 \pm 0.0028$ & $2454506.365 \pm 0.0030$ \\
$K_{1}\left(\mathrm{~km} \mathrm{~s}^{-1}\right)$ & $43.20 \pm 0.18$ & $43.504 \pm 0.082$ \\
$K_{2}\left(\mathrm{~km} \mathrm{~s}^{-1}\right)$ & $46.39 \pm 0.18$ & $46.438 \pm 0.101$ \\
$e$ & 0.0 (adopted) & 0.0 (adopted) \\
$\gamma\left(\mathrm{km} \mathrm{s}^{-1}\right)$ & $-12.43 \pm 0.11$ & $-12.405 \pm 0.051$ \\
$m_{1} \sin ^{3} i\left(M_{\odot}\right)$ & $0.1332 \pm 0.0012$ & $0.13313^{\mathrm{b}} \pm 0.00064$ \\
$m_{2} \sin ^{3} i\left(M_{\odot}\right)$ & $0.1231 \pm 0.0011$ & $0.12472^{\mathrm{b}} \pm 0.00054$ \\
$a_{1} \sin i\left(10^{6} \mathrm{~km}\right)$ & $2.0317 \pm 0.0083$ & $2.0460 \pm 0.0039$ \\
$a_{2} \sin i\left(10^{6} \mathrm{~km}\right)$ & $2.1816 \pm 0.0087$ & $2.1840 \pm 0.0047$ \\
Standard error of a unit weight observation $\left(\mathrm{km} \mathrm{s}^{-1}\right)$ & 0.6 & 0.4 \\
\hline
\end{tabular}

Notes.

a $T_{0}$ is a time of maximum velocity of the primary.

b Minimum masses computed with the physical constants and formula recommended by Torres et al. (2010).

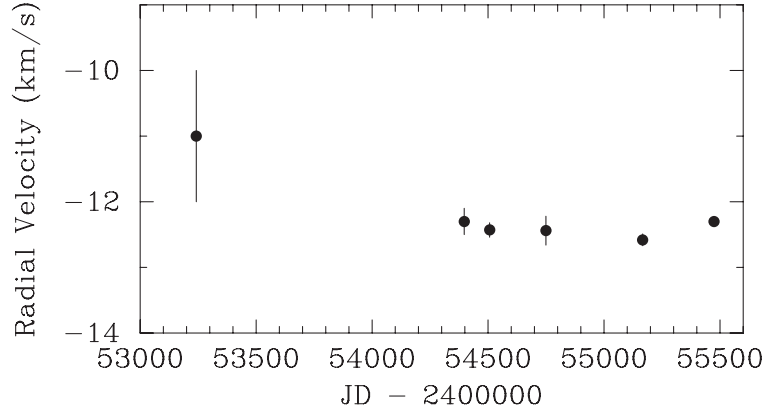

Figure 2. Center-of-mass radial velocities and their uncertainties at six epochs plotted vs. Julian date.

The newly determined center-of-mass velocity from the MIKE spectra of Weinberger (2008) is also in excellent agreement. The single velocity of Song et al. (2005) is a measurement of blended component lines that are somewhat unequal in strength and so may be a bit biased. Nevertheless, it is consistent with our weighted average systemic velocity. Thus, the radial velocities, acquired to date, provide no evidence for orbital motion about a third body.

\section{SHORT-PERIOD SPECTROSCOPIC ORBIT}

Our center-of-mass velocities for the past four seasons (Table 2) show no evidence of any significant velocity change. Thus, we have used our individual velocities, measured from the Fairborn Observatory, KPNO, and Lick Observatory spectrograms and weighted as discussed above, to obtain an improved circular orbit for the 3.4 day binary. Table 4 compares our new circular orbital elements and related parameters to the earlier one of Zuckerman et al. (2008). For a circular orbit the element $T$, a time of periastron passage, is undefined. So as recommended by Batten et al. (1989), $T_{0}$, a time of maximum velocity of the primary, is given instead. The uncertainties of most of the parameters are improved by a factor of two or more. In Figure 3, the observed velocities are compared with the computed velocity curves. For completeness sake, we mention that allowing the eccentricity to vary results in a solution with an extremely small eccentricity value of $0.0058 \pm 0.0019$.

\section{ABUNDANCE ANALYSIS}

We analyzed the Lick Observatory echelle spectrum of BD $+20^{\circ} 307$ that was obtained on 2008 August 14, which has a

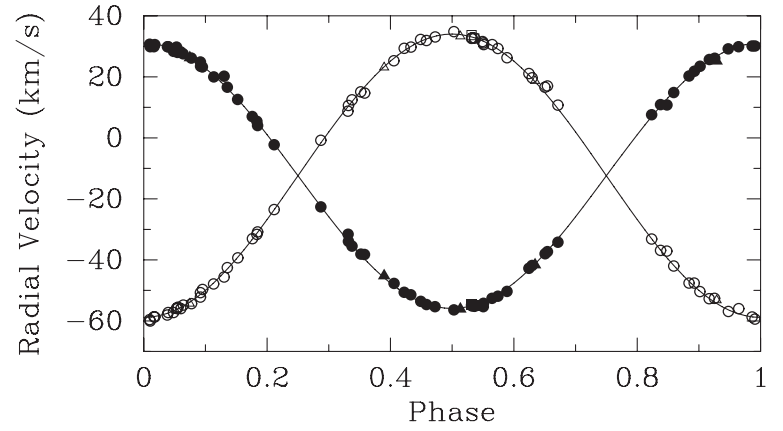

Figure 3. Radial velocities compared with the computed radial velocity curve of $\mathrm{BD}+20^{\circ} 307$. Filled and open symbols represent the primary and secondary, respectively. Circles: Fairborn Observatory; triangles: KPNO; squares: Lick Observatory. Zero phase is a time of maximum positive velocity of the primary ( $T_{0}$ in Table 4).

signal-to-noise ratio of typically 120-200 per pixel, depending on wavelength. Lines were selected for measurement in the wavelength region 5000-7000 $\AA$. BD $+20^{\circ} 307$ was observed near quadrature with a velocity difference of $\sim 87 \mathrm{~km} \mathrm{~s}^{-1}$, which leads to blending of many primary and secondary features. Therefore, the line lists for the primary and secondary stars differ. Lines that overlap with stellar features of the companion star, lines that are strongly blended within the spectrum of a single star, and lines that do not lie on the linear part of the curve of growth (reduced width $>-4.5$ after correction) were not considered in our line lists. The lists include $\sim 40 \mathrm{Fe}$ I lines and between 1 and 21 lines for the other elements. The excitation potentials (EPs) were taken from Thevenin (1989, 1990), and $\log g f$ values were estimated from Procyon (F5V), which has an effective temperature that is close to those of the stars considered in this study. Equivalent widths (EWs) were measured interactively with the IRAF splot package, in which multiple Gaussian functions can be used to account for possible blends. Because the EWs were determined relative to the combined continuum, the measured EWs must be corrected to the true stellar continuum with the ratio of the fluxes of the two stars. The flux ratio found by Weinberger (2008) yields correction factors of 1.709 and 2.387 for the primary and secondary, respectively. Table 5 summarizes the information for the lines that we have used. The full list is available online. That table identifies the specific lines measured, giving for each the element and ionization stage plus the wavelength, as well as the EP, oscillator strength $\log g f$ value, and our corrected EW. 


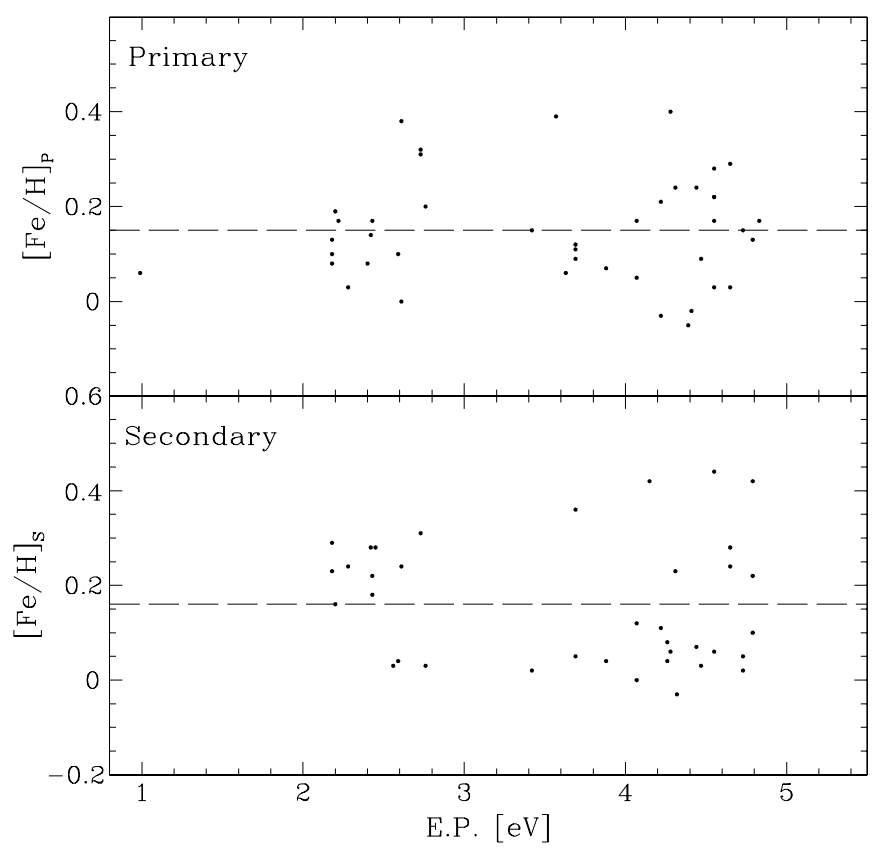

Figure 4. Top and bottom panels for the primary and secondary components, respectively, show metallicity vs. excitation potential. The dashed line in the two panels indicates the adopted $[\mathrm{Fe} / \mathrm{H}]$ value for each star.

Table 5

Equivalent Widths

\begin{tabular}{lccccc}
\hline \hline Element & $\begin{array}{c}\text { Wavelength } \\
(\AA)\end{array}$ & $\begin{array}{c}\mathrm{EP} \\
(\mathrm{eV})\end{array}$ & $\log g f$ & $\begin{array}{c}\mathrm{EW}_{1} \\
(\mathrm{m \AA})\end{array}$ & $\begin{array}{c}\mathrm{EW}_{2} \\
(\mathrm{~m} \AA)\end{array}$ \\
\hline $\mathrm{Na}$ I & 5688.22 & 2.10 & -0.24 & $\ldots$ & 99.3 \\
$\mathrm{Mg}$ I & 5711.10 & 4.34 & -1.78 & $\ldots$ & 109.3 \\
$\mathrm{Si}$ I & 5701.11 & 4.93 & -2.06 & $\ldots$ & 28.43 \\
$\mathrm{Si}$ I & 5708.41 & 4.95 & -1.39 & $\ldots$ & 81.4 \\
$\mathrm{Si}$ I & 5772.15 & 5.08 & -1.69 & 50.2 & 51.3 \\
$\mathrm{Si}$ I & 5793.08 & 4.93 & -1.80 & 33.8 & 37.0 \\
$\mathrm{Si}$ I & 5797.87 & 4.95 & -1.95 & $\ldots$ & 30.8 \\
$\mathrm{Si}$ I & 6131.58 & 5.61 & -1.76 & 22.2 & 21.2 \\
$\mathrm{Si}$ I & 6131.87 & 5.61 & -1.66 & $\ldots$ & 16.2 \\
\hline
\end{tabular}

(This table is available in its entirety in a machine-readable form in the online journal. A portion is shown here for guidance regarding its form and content.)

\subsection{Model Atmosphere Parameters}

The local thermodynamic equilibrium (LTE) model stellar atmospheres were obtained from interpolation within the ATLAS9 (Castelli et al. 1997) grid of non-overshoot models with an interpolation program provided by I. Ivans (2004, private communication). The initial models were generated with a solar metallicity as a starting point, but once the Fe I abundance was determined, a new model was generated with a metallicity of $[\mathrm{m} / \mathrm{H}]=0.10$.

The effective temperatures for the primary and secondary (6500 and $6250 \mathrm{~K}$, respectively) were taken from Weinberger (2008), who compared the spectrum of BD $+20^{\circ} 307$ with synthetic spectra. Given masses between 1.2 and $1.3 M_{\odot}$ (Weinberger 2008) and the above effective temperatures, we adopted a surface gravity of $\log g=4.60$ and 4.75 for the primary and secondary stars, respectively. The temperature values are confirmed in Figure 4, which shows the derived $[\mathrm{Fe} / \mathrm{H}]$ abundance from each Fe I line measured in the spectrum. The absence of any dependence of derived abundance on EP indicates that the adopted temperatures are appropri-
Table 6

Atmospheric Parameters

\begin{tabular}{lcccc}
\hline \hline Component & $\begin{array}{l}T_{\text {eff }} \\
(\mathrm{K})\end{array}$ & $\log g$ & {$[\mathrm{Fe} / \mathrm{H}]$} & $\begin{array}{c}v_{t} \\
\left(\mathrm{~km} \mathrm{~s}^{-1}\right)\end{array}$ \\
\hline Primary & 6500 & 4.60 & 0.1 & 1.65 \\
Secondary & 6250 & 4.75 & 0.1 & 1.50 \\
\hline
\end{tabular}

ate for both the primary and secondary components. Our adopted surface gravities differ from the value $\log g=5.0$ that was adopted by Weinberger (2008) but provide a good match between abundances derived from $\mathrm{Fe}_{\mathrm{I}}$ and $\mathrm{Fe}$ II lines. We estimated microturbulence velocities of $v_{t}=1.65$ and $1.50 \mathrm{~km} \mathrm{~s}^{-1}$ for the primary and secondary stars, respectively, that result in no trend between the derived Fe I abundance and the reduced EW. Atmospheric parameters are summarized in Table 6.

\subsection{Abundance Determinations}

Detailed model atmosphere analysis was used to determine chemical abundances for $\mathrm{Na}, \mathrm{Mg}, \mathrm{Si}, \mathrm{Ca}, \mathrm{Ti}, \mathrm{Cr}, \mathrm{Mn}, \mathrm{Fe}, \mathrm{Co}$, and $\mathrm{Ni}$ with the abfind driver in the LTE spectral analysis code MOOG (Sneden 1973, 2010 version). Abundances have been determined relative to Procyon, adopting $\log g f$ values that reproduce the solar abundances of this star. EWs were measured from the Procyon Atlas (Griffin 1979), and the non-overshoot Kurucz atmosphere model was generated with the following stellar parameters: $T_{\text {eff }}=6500 \mathrm{~K}, \log g=4.00,[\mathrm{Fe} / \mathrm{H}]=0.00$, and $v_{t}=2.1 \mathrm{~km} \mathrm{~s}^{-1}$, which are in agreement with Steffen's (1985) values. The systematic difference between our adopted $\log g f$ values and the values presented in Thevenin $(1989,1990)$ is only $0.03 \mathrm{dex}$ (in the sense (this paper - Thevenin)) with an rms of 0.17 dex.

Li I abundances were determined by comparing the observed spectrum with a synthetic spectrum, which consists of a combination of both primary and secondary stellar synthetic spectra, that was created with the binary driver (MOOG; 2010 version). The line list for the Li I $\lambda 6707$ feature, which is a doublet with both isotopic and hyperfine splitting that is blended with an Fe I line, was taken from Reddy et al. (2002).

\subsection{Results and Error Analysis}

Table 7 presents our LTE abundances relative to Procyon for both components. Also listed for the primary and secondary is the number of lines used for each element and ionization stage. Because of blending problems, we were unable to determine abundances for $\mathrm{NaI}$ and $\mathrm{Mg}$ I in the primary and $\mathrm{Cr}$ II in the secondary. In the final column, a mean of the primary and secondary $\sigma$ values is listed. This is a representative value for the uncertainty in a given abundance determination. The metallicity of both stars appears to be slightly supersolar, with Fe abundances for the primary and secondary stars of $[\mathrm{Fe} / \mathrm{H}]$ $=0.15 \pm 0.11$ and $0.16 \pm 0.13$, respectively, based on the strong agreement of 43 and $37 \mathrm{Fe}$ I lines. The total uncertainty in the metallicity is calculated as described below. Additionally, the abundances of most other measured species are either solar or slightly supersolar. The $\mathrm{Cr}$ I and $\mathrm{Cr}$ II abundances and $\mathrm{Ti}$ I and Ti II abundances for the primary star show an unexpected difference, but in both cases the abundances are based on only two or three lines. With the exception of these two anomalous results, the abundances of most other measured species are in excellent agreement between both stars, supporting the adopted stellar atmosphere parameters. 


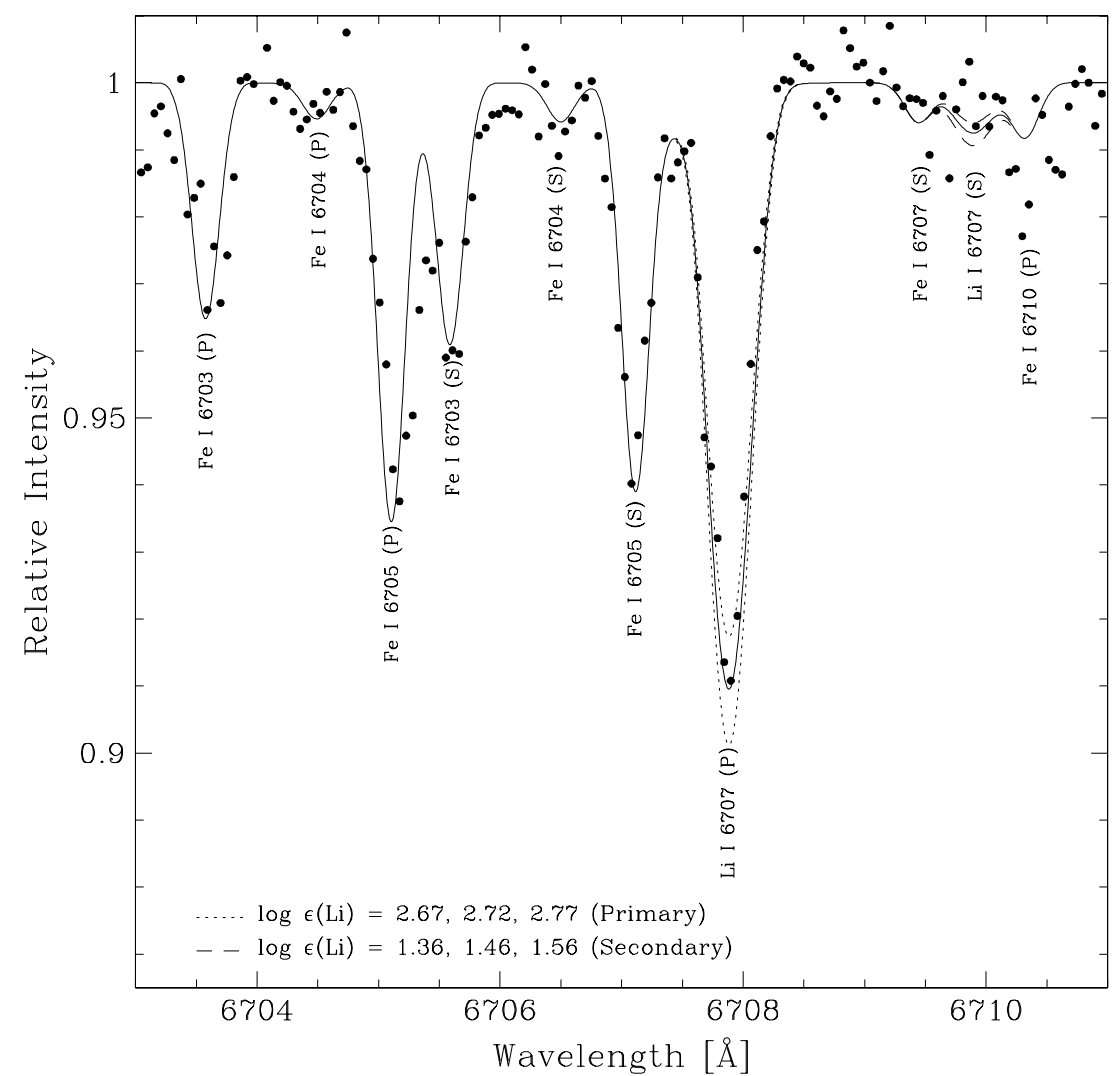

Figure 5. Spectrum synthesis of the Li region and the Li I $\lambda 6707$ features. The solid line shows the best fit for the Li abundances of the primary and secondary stars, $\log \epsilon(\mathrm{Li})=2.72$ and 1.46, respectively. Because of the weakness of the secondary line, we consider the latter value to be an upper limit. The dotted lines show fits that differ by \pm 0.05 dex from the adopted abundance fit to the primary line, while the dashed lines differ by \pm 0.1 dex from the adopted fit to the secondary.

Table 7

LTE Abundances of the Primary and Secondary

\begin{tabular}{|c|c|c|c|c|c|}
\hline Element & $\begin{array}{c}\text { Primary } \\
{[\mathrm{x} / \mathrm{H}]}\end{array}$ & $\begin{array}{l}\text { Number } \\
\text { of Lines }\end{array}$ & $\begin{array}{c}\text { Secondary } \\
{[\mathrm{x} / \mathrm{H}]}\end{array}$ & $\begin{array}{l}\text { Number } \\
\text { of Lines }\end{array}$ & $\sigma$ \\
\hline $\mathrm{Na} I$ & .. & 0 & -0.48 & 1 & 0.13 \\
\hline $\mathrm{Mg}_{\mathrm{I}}$ & ... & 0 & 0.20 & 1 & 0.13 \\
\hline Si I & -0.02 & 7 & -0.03 & 9 & 0.15 \\
\hline Ca I & 0.14 & 5 & 0.13 & 7 & 0.12 \\
\hline Ti I & 0.36 & 3 & 0.24 & 6 & 0.20 \\
\hline Ti II & -0.03 & 2 & 0.19 & 2 & 0.12 \\
\hline CrI & 0.33 & 3 & 0.38 & 3 & 0.12 \\
\hline Cr II & -0.01 & 3 & $\ldots$ & 0 & 0.11 \\
\hline Mn I & 0.20 & 3 & 0.22 & 3 & 0.12 \\
\hline $\mathrm{Fe} I$ & 0.15 & 43 & 0.16 & 37 & 0.12 \\
\hline $\mathrm{Fe}$ II & 0.14 & 4 & 0.14 & 8 & 0.13 \\
\hline Co I & 0.21 & 1 & -0.07 & 1 & 0.12 \\
\hline Ni I & -0.02 & 15 & 0.07 & 21 & 0.15 \\
\hline Li I & $2.72^{\mathrm{a}}$ & 1 & $\leqslant 1.46^{\mathrm{a}}$ & 1 & 0.12 \\
\hline
\end{tabular}

Note. ${ }^{\text {a }}$ For lithium, the quantity shown is $\log \epsilon(\mathrm{Li})$.

Weinberger (2008) noted the difference in the Li I $\lambda 6707$ line strength in the two components of the binary, with a relatively strong feature in the primary spectrum and a limit of $<6 \mathrm{~mA}$ in the secondary spectrum. Spectral synthesis of the $\mathrm{Li} \lambda 6707$ region (Figure 5) indicates that the $\mathrm{Li}$ abundance of the primary star is $\log \epsilon(\mathrm{Li})=2.72$, and the upper limit to the $\mathrm{Li}$ abundance in the secondary is $\log \epsilon(\mathrm{Li})=1.46$. The lithium abundances are also given in Table 7.

Uncertainties due to errors in atmospheric parameters were estimated by increasing $T_{\text {eff }}, \log g$, and $v_{t}$, one parameter at a time, while the other two parameters were kept fixed, and the
Table 8

Abundance Uncertainties due to Atmospheric Parameters

\begin{tabular}{|c|c|c|c|}
\hline Element & $\begin{array}{l}\Delta T_{\text {eff }}= \\
+100 \mathrm{~K}\end{array}$ & $\begin{array}{c}\Delta \log g= \\
\quad+0.3\end{array}$ & $\begin{array}{c}\Delta v_{t}= \\
+0.3 \mathrm{~km} \mathrm{~s}^{-1}\end{array}$ \\
\hline $\mathrm{NaI}$ & -0.05 & 0.05 & 0.02 \\
\hline $\mathrm{Mg}_{\mathrm{I}}$ & -0.06 & 0.06 & 0.04 \\
\hline Si I & -0.03 & 0.01 & 0.15 \\
\hline Ca I & -0.06 & 0.05 & 0.06 \\
\hline Ti I & -0.07 & -0.01 & 0.04 \\
\hline Ti II & -0.01 & 0.07 & 0.10 \\
\hline $\mathrm{Cr} \mathrm{I}$ & -0.06 & 0.01 & 0.05 \\
\hline Cr II & 0.12 & -0.06 & 0.06 \\
\hline Mn I & -0.06 & 0.01 & 0.06 \\
\hline $\mathrm{Fe} I$ & -0.06 & 0.03 & 0.07 \\
\hline Fe II & 0.01 & -0.06 & 0.08 \\
\hline CoI & -0.06 & 0.00 & 0.03 \\
\hline $\mathrm{Ni} I$ & -0.06 & -0.01 & 0.04 \\
\hline
\end{tabular}

total uncertainty was obtained adding individual uncertainties in quadrature. The uncertainty in $T_{\text {eff }}$ is estimated to be $100 \mathrm{~K}$ based on changes with temperature in the slope of the derived abundance versus EP plot. The uncertainty for $\log g$ in both stars was obtained by modifying its value until the $\mathrm{Fe}_{\mathrm{I}}$ and Fe II abundances differed by 0.10 dex; the uncertainty for $v_{t}$ was obtained by modifying its value until the $\mathrm{Fe}$ I abundance differed by 0.1 dex between typical weak and strong lines. Table 8 presents the individual model dependencies for each element typical of both stars. For some species, the uncertainty in $T_{\text {eff }}$ dominates the total uncertainty, while uncertainties for other species are dominated by errors in $\log g$, microturbulence, or 
Table 9

Synthesis Analysis Uncertainties of the Lithium Abundances

\begin{tabular}{lcc}
\hline \hline Parameter & \multicolumn{2}{c}{ Abundance Uncertainty } \\
\cline { 2 - 3 } & Primary & Secondary \\
\hline$T_{\mathrm{eff}}{ }^{\mathrm{a}}$ & 0.07 & 0.10 \\
$\log g^{\mathrm{a}}$ & 0.01 & 0.01 \\
$v_{t}{ }^{\mathrm{a}}$ & 0.01 & 0.08 \\
Fitting uncertainty & 0.01 & 0.05 \\
Smoothing uncertainty & 0.02 & 0.04 \\
Continuum uncertainty & 0.01 & 0.05 \\
Total & 0.08 & 0.15 \\
\hline
\end{tabular}

Note. ${ }^{\mathrm{a}}$ Uncertainty results from the change of parameter given in Table 8.

line measurements. The uncertainty in Fe I is typically $\sim 0.1 \mathrm{dex}$ for both stars.

Fe I is a minority species in stellar atmospheres with $T_{\text {eff }}>$ $4500 \mathrm{~K}$, and it can deviate from LTE due to overionization, which means that non-LTE (NLTE) abundance corrections can be important. Fortunately, according to Mashonkina et al. (2011), NLTE corrections for Fe I are small $(<0.1$ dex $)$ and for Fe II are insignificant $(\sim 0.02$ dex $)$ in solar metallicity stars. Therefore, our iron abundance determinations are not affected by LTE departures.

The total uncertainty for each $\mathrm{Li}$ abundance depends on the uncertainties in atmospheric parameters and the uncertainties related to matching the synthetic spectrum to the observed one. The sources for the latter are fitting, smoothing, and continuum uncertainties. The fitting uncertainty is identified as the minimum noticeable difference that appears when the $\mathrm{Li}$ abundance is modified after the best fit that matches the observed spectrum is obtained. The smoothing uncertainty arises when matching the resolution of the data by smoothing the synthetic spectrum with Gaussians. The FWHM measurement of the synthetic spectrum has an uncertainty of 0.03 , which propagates into the total abundance uncertainty. The continuum uncertainty arises from the uncertainty in the placement of the continuum. For both components, Table 9 lists each individual uncertainty and the final addition in quadrature of those values, the total uncertainty.

\section{DISCUSSION}

\subsection{Third Component}

Our near-infrared AO image shows no evidence of a third component at separations of 19 AU or greater. Our radial velocity data, which are sensitive to smaller separations out to perhaps $5 \mathrm{AU}$, likewise give no evidence for the existence of a third component. However, because the separations covered by the two types of data do not overlap, a third body orbiting the short-period binary might have been missed. Thus, using Kepler's third law, we explore the kinds of long-period orbits that could have avoided detection by our observations to date. We first estimate the total mass of the possible three-body system because for a given period of the supposed long-period orbit, the mass of the third component has only a modest effect on the long-period separation. The temperature of the shortperiod primary corresponds to a dwarf of mass $1.3 M_{\odot}$ (Table B1, Gray 1992), which with our mass ratio produces a mass of $1.22 M_{\odot}$ for the secondary, making a total binary mass of $2.52 M_{\odot}$, the minimum for a possible triple system. A mass of $0.9 M_{\odot}$ corresponds to a late-G dwarf and is the upper limit to
Table 10

Long-period Orbits Conjectured for Total Mass 2.62 $M_{\odot}$

\begin{tabular}{|c|c|c|c|c|c|c|c|}
\hline \multirow{2}{*}{$\begin{array}{l}\text { Orbital } \\
\text { Period } \\
(\mathrm{yr})\end{array}$} & \multirow{2}{*}{$\begin{array}{c}a_{L}^{\mathrm{a}} \\
(\mathrm{AU})\end{array}$} & \multicolumn{3}{|c|}{ Periastron } & \multicolumn{3}{|c|}{ Apastron } \\
\hline & & $\begin{array}{c}e=0.3 \\
(\mathrm{AU})\end{array}$ & $\begin{array}{c}e=0.5 \\
(\mathrm{AU})\end{array}$ & $\begin{array}{c}e=0.9 \\
(\mathrm{AU})\end{array}$ & $\begin{array}{c}e=0.3 \\
(\mathrm{AU})\end{array}$ & $\begin{array}{c}e=0.5 \\
(\mathrm{AU})\end{array}$ & $\begin{array}{c}e=0.9 \\
(\mathrm{AU})\end{array}$ \\
\hline 5 & 4.0 & 2.8 & 2.0 & 0.4 & 5.2 & 6.0 & 7.6 \\
\hline 10 & 6.4 & 4.5 & 3.2 & 0.6 & 8.3 & 9.6 & 12.2 \\
\hline 50 & 18.7 & 13.1 & 9.4 & 1.9 & 24.3 & 28.0 & 35.5 \\
\hline 100 & 29.7 & 20.8 & 14.8 & 3.0 & 38.6 & 44.6 & 56.4 \\
\hline 500 & 86.8 & 60.8 & 43.4 & 8.7 & 112.8 & 130.2 & 164.9 \\
\hline
\end{tabular}

Note. ${ }^{\mathrm{a}} a_{L}$ is the semimajor axis of the long-period orbit.

the mass of a supposed third component because such a star would be bright enough for us to detect its absorption lines in our spectra. While increasing the mass of the third component from 0.01 to $0.9 M_{\odot}$ raises the total mass of the proposed triple system from 2.53 to $3.42 M_{\odot}$, this change results in only an $11 \%$ increase in the semimajor axis of the long-period orbit. For a representative set of orbital solutions, we adopt a third component mass, $M_{3}$, of $0.1 M_{\odot}$, which makes the system's total mass $2.62 M_{\odot}$. Varying the orbital period from 5 to $500 \mathrm{yr}$ then produces the semimajor axes of the long-period orbits, $a_{L}$, and the associated apastron and periastron distances for specific eccentricity values of $0.3,0.5$, and 0.9 , all of which are summarized in Table 10.

Zuckerman et al. (2008) explained the unusually high infrared flux of $\mathrm{BD}+20^{\circ} 307$ as an extremely large amount of warm dust and interpreted it as the aftermath of a recent collision of two planet-mass objects. Recently, Weinberger et al. (2011) estimated a lifetime of $\sim 80,000 \mathrm{yr}$ for the dust. They also attempted to match a Spitzer 5-37 $\mu \mathrm{m}$ infrared spectrum of BD $+20^{\circ} 307$ with a model of dust that is composed of four types of particulates. The adopted fit adequately represents the spectrum and most of its major emission features if the dust is situated at a distance of $0.85 \mathrm{AU}$ from the center of the binary orbit. This distance is much larger than the close binary separation of 0.06 AU (Weinberger 2008). From Table 10, a 10 yr long-period orbit with an eccentricity of 0.9 has a periastron separation of $0.6 \mathrm{AU}$, a value that is similar to the semimajor axis of the dust particles that result from the rocky body collision(s) in the system. Such a close encounter makes it difficult to believe that planets could have stable orbits over the $\geqslant 1 \mathrm{Gyr}$ lifetime of the system. Smaller eccentricities of 0.5 and 0.3 increase the periastron distance to 3.2 and $4.5 \mathrm{AU}$, respectively, corresponding to distances from the binary that are only 4-5 times greater than that of the dust. These results and the four seasons of constant center-of-mass velocity suggest that any long-period orbit likely has a period greater than $10 \mathrm{yr}$. For a period of $50 \mathrm{yr}$, the semimajor axis is similar to the minimum separation detectable in our near-infrared AO image, while for most $500 \mathrm{yr}$ orbits, the separations are so large that a possible third component should have been detected. While we have found no evidence of a third component, these results indicate that our current combination of radial velocities and imaging cannot eliminate most orbits with periods ranging from 10 to perhaps a few hundred years.

\subsection{Age of the $B D+20^{\circ} 307$ System}

Because $\mathrm{BD}+20^{\circ} 307$ is a short-period binary, it is not possible to use its rotation period or X-ray flux to estimate the age of the system because both are compromised by the forced 
rapid rotation of the close binary. The lithium abundances of the components, compared to those of stars in open clusters of known age, are another potential way to estimate the system's age. This is because in stars with convective outer atmospheres the surface lithium is moved to the stellar interior where it is destroyed at relatively low temperatures. However, various studies (for a review, see Randich 2010) have shown that lithium depletion in late-type stars is not just a function of age and mass but depends on at least one other parameter. Stellar models that include additional physics such as rotationally induced mixing, magnetic fields, gravity waves, and angular momentum redistribution have been developed (see, e.g., Sestito \& Randich 2005; Schuler et al. 2011), but none have been completely successful in explaining the full variety of lithium abundances.

Based on the effective temperatures and $\log g$ values of our abundance analysis, the short-period binary components are a mid- and a late-F dwarf (e.g., Table B1, Gray 1992) that differ in temperature by only $250 \mathrm{~K}$. The stars have comparable masses (mass ratio of 0.94$)$, rotational velocities ( $v \sin i=10 \mathrm{~km} \mathrm{~s}^{-1}$; Weinberger 2008), and abundances (Table 7). Because of the similarities of the two components, we would expect their lithium abundances to be nearly alike as well, unless the hotter star is in the "lithium dip," the name given to a large decrease in the lithium abundance that occurs in a very narrow temperature range, corresponding to early- and mid-F dwarfs. Instead, in BD $+20^{\circ} 307$ it is the lithium abundance of the late-F secondary that is very low.

The system's components avoid the pitfall of being in the center of the lithium dip, which is centered at about $6650 \mathrm{~K}$ for stars in the Hyades cluster (Boesgaard \& Tripicco 1986). This relatively young cluster has an age of $0.625 \mathrm{Gyr}$ (Perryman et al. 1998) and an average iron abundance of $[\mathrm{Fe} / \mathrm{H}]=0.13$ (Paulson et al. 2003), which makes it somewhat iron rich relative to the Sun but very similar to the components of BD $+20^{\circ} 307$. The temperature of $\mathrm{BD}+20^{\circ} 307$ 's primary component lies on the cool side of the lithium dip, and its abundance of $\log \epsilon$ $(\mathrm{Li})=2.72$ is consistent with the abundances of Hyades stars at the same temperature and mass but also not much greater than the value for the intermediate-age (1.8 Gyr; Daniel et al. 1994) open cluster NGC 752 (Balachandran 1995; Anthony-Twarog et al. 2009). Sestito et al. (2004) have concluded that metallicity variations of about \pm 0.2 do not affect lithium depletion for clusters older than the Hyades.

Several open cluster studies (Thorburn et al. 1993; Barrado y Navascués \& Stauffer 1996; Barrado y Navascués et al. 1997) have shown that synchronously rotating short-period binaries have lithium abundances that are significantly above the mean trend for single stars. These results suggest that the lithium abundances of $\mathrm{BD}+20^{\circ} 307$ are enhanced. If true, then the abundance of the primary argues for an age greater than that of the Hyades cluster and probably at least 1 Gyr old.

The secondary star is just $250 \mathrm{~K}$ cooler than the primary and lies in the temperature range between the lithium dip and the region of classic lithium depletion that begins in early-G dwarfs. In the late-F dwarf temperature range, the lithium abundance remains relatively high, even in old open clusters (e.g., Sestito \& Randich 2005). If the primary's lithium abundance is enhanced, that of the secondary would be expected to be similar or perhaps even larger in value because that star is situated completely outside the lithium dip region. For example, at the temperature of the secondary, close binaries in the Hyades have $\log \epsilon(\mathrm{Li})>3.0$ (Barrado y Navascués \& Stauffer 1996). However, instead of a similar abundance, the lithium line of the secondary is so weak that we have only been able to determine an upper limit of $\log \epsilon$ $(\mathrm{Li})=1.46$. Such a low lithium abundance in the secondary is well below the values observed in stars of comparable temperature in NGC 752 and even M67 (age = 4 Gyr; Demarque et al. 1992), which have lithium abundances of about $\log \epsilon(\mathrm{Li})=$ 2.65 and 1.9, respectively (Sestito \& Randich 2005). The value for M67 is for its lithium-poor stars as discussed below. Thus, even an advanced age cannot completely explain the low lithium abundance in the secondary star.

While late-type main-sequence stars in many well-studied open clusters (e.g., the Hyades, NGC 752, NGC 3680) show a tight correlation between lithium abundance and effective temperature or its proxies such as $B-V$ or mass, the stars in a small number of clusters, of which M67 is perhaps the beststudied example, show a significant spread in lithium at a given temperature. In the case of M67, 30\%-40\% of the analyzed stars are lithium poor by a factor of 10 or more relative to the rest, the latter stars being characterized as lithium rich (Pasquini et al. 1997; Jones et al. 1999). However, even when compared to the lithium-poor stars of similar temperature in M67, the lithium abundance upper limit of BD $+20^{\circ} 307$ 's secondary is unusual in its low value. NGC 6253, having an age of $3 \mathrm{Gyr}$, is the youngest cluster with a significant number of lithium-poor stars (Randich 2009). Thus, if the lithium abundance upper limit of the secondary is taken at face value, it suggests that BD $+20^{\circ} 307$ may be $3 \mathrm{Gyr}$ or older. Indeed, with an upper limit of 1.46 the secondary's lithium abundance may be similar to that of the Sun, which has $\log \epsilon(\mathrm{Li})=1.1$ (Grevesse \& Sauval 1998).

From a study of numerous open clusters, Randich (2009) has concluded that a high lithium abundance provides only a lower limit to a star's age, while a low lithium abundance, similar to that of the Sun, denotes an old age and in some cases indicates that another parameter besides age and mass enhances the lithium depletion. Although a specific age cannot be assigned to $\mathrm{BD}+20^{\circ} 307$, it is likely to range from one to several Gyr, as suggested by both Weinberger (2008) and Zuckerman et al. (2008), and the system may even be as old as the Sun.

Zuckerman et al. (2008) argued that the extensive amount of warm dust in the $\mathrm{BD}+20^{\circ} 307$ system does not result from a very young star beginning the process of making planets, but rather comes from the recent collision of asteroids or perhaps planetsized objects in a relatively mature main-sequence system. Several studies (e.g., Israelian et al. 2009; Gonzalez et al. 2010) have concluded that among solar analogs, stars with planets have enhanced lithium depletion compared to similar temperature stars without planetary systems. A system that would seem to support this claim is the solar-like pair $16 \mathrm{Cyg} \mathrm{A}$ and B, which have a common proper motion. Component B hosts a giant planet (Cochran et al. 1997), while component A has no detectable planetary companion. A recent detailed abundance analysis of the pair (Schuler et al. 2011) found the two stars to differ by only $43 \mathrm{~K}$ in effective temperature and to have essentially identical abundances for 15 elements. However, the lithium abundances of these two stars differ by a factor of at least 4.5. Schuler et al. (2011) concluded that the rather different lithium abundances in the two very similar stars likely were caused by rotationally induced mixing and differences in angular momentum history and noted the theoretical work of Bouvier (2008) in support of their scenario. The formation of a massive planet in 16 Cyg B requires a disk with a lifetime long enough to support planet formation. This would allow more time for star-disk interactions to slow the star, and according 
to Bouvier's theory, slow rotators are expected to deplete more lithium than fast rotators.

While this result might lend credence to the very low lithium abundance of the $\mathrm{BD}+20^{\circ} 307$ secondary, it does not explain the much higher abundance of the primary. Although the components of $\mathrm{BD}+20^{\circ} 307$ are hotter and have a larger temperature difference than $16 \mathrm{Cyg} \mathrm{A}$ and $\mathrm{B}$, the short orbital period of 3.4 days for $\mathrm{BD}+20^{\circ} 307$ argues that any circumstellar disk would have to be circumbinary, and so any planetary system would have to orbit both stars, as appears to have been the case (Song et al. 2005). Its effects, as discussed above, should be similar for the two components. However, in the case of BD $+20^{\circ} 307$, the stars differ in lithium abundance by a factor of at least 18 , much greater than that of $16 \mathrm{Cyg} \mathrm{A}$ and $\mathrm{B}$. We also note that the claim of lower lithium abundances in planet-host stars has been countered by other studies (e.g. Luck \& Heiter 2006; Baumann et al. 2010) that have concluded that there is no difference in the lithium abundance trends for the two groups of stars, those with and those without planets. Thus, there is no consensus on the observational results needed to support the theory.

As discussed by Thorburn et al. (1993) and Barrado y Navascués et al. (1997), obtaining lithium abundances of double-lined binaries is a much more complicated process than that for single stars because of the presence of the secondary's spectrum; thus, the lithium abundances of the components in such binaries are not often determined. Perhaps the most extensive analysis of lithium abundances in double-lined systems is that of Barrado y Navascués et al. (1997), who examined 41 dwarf and subgiant chromospherically active binaries. Most were single lined, but in their sample we identify three doublelined short-period binaries with F-and/or G-dwarf components, making them similar to $\mathrm{BD}+20^{\circ} 307$. However, unlike BD $+20^{\circ} 307$, in each of those cases the two binary components have similar lithium abundances.

Although a field star with at best a poorly determined age, BD $+20^{\circ} 307$ is a short-period, synchronously rotating binary with coeval stars. Thus, the extremely different lithium abundances in very similar components with relatively thin outer convection zones put significant constraints on any third parameter that affects those abundances. Although various suggestions as to the nature of the additional parameter have been offered, it has so far defied identification despite a substantial increase in the number of open clusters with measured lithium abundances (Randich 2010) that are available for comparison with theory.

\section{CONCLUSIONS}

With AO imaging and high-resolution spectroscopy we searched for a tertiary companion of the dusty close binary BD $+20^{\circ} 307$. As discussed in earlier papers, the warm dust is likely the aftermath of the collision of rocky bodies in the terrestrial planet zone around the 3.4 day binary. For separations of $19 \mathrm{AU}$ and greater, there is no evidence for a tertiary companion in the AO image. The brightness contrast is such that we can eliminate a third star with a mass as low as $0.1 M_{\odot}$ at projected separations of $45 \mathrm{AU}$, while at separations greater than $\sim 200 \mathrm{AU}$ even companions below 40-60 Jupiter masses are eliminated. Our radial velocities, covering four seasons, also produce no evidence for a third component, and with them we have determined an improved circular orbit with a period of 3.42008 days. Earlier velocity observations at two different epochs also are consistent with our systemic velocity. However, the separations covered by our imaging and radial velocities do not overlap.
Thus, using Kepler's third law, we have explored the parameter space to determine what kind of long-period orbits might have avoided detection by our observations to date. Given that the observable separation may be foreshortened because of the orientation of the orbit, our combination of radial velocities and imaging cannot eliminate most orbits with periods ranging from 10 to perhaps a few hundred years. Confirmation or rejection of a possible third component in that period range will require more sensitive future AO observations and additional seasons of radial velocity monitoring.

From an abundance analysis of an echelle spectrum, the shortperiod mid-F dwarf primary and late-F dwarf secondary have $[\mathrm{Fe} / \mathrm{H}]=0.15$ and 0.16 , respectively, and so are somewhat metal-rich relative to the Sun. Those values are very similar to iron abundances found for Hyades cluster stars. The lithium abundance of the primary is $\log \epsilon(\mathrm{Li})=2.72$, while that of the secondary is an upper limit, $\log \epsilon(\mathrm{Li})=1.46$. When compared to the lithium abundances of stars in open clusters of known age, the values do not produce a consistent age but suggest that the system is at least 1 Gyr old and could be as old as the Sun.

We thank Christian Marois for help and discussions concerning the Keck AO images and Caty Pilochowski for her guidance of the abundance analysis. Some of the data presented herein were obtained at the W. M. Keck Observatory, which is operated as a scientific partnership among the California Institute of Technology, the University of California, and the National Aeronautics and Space Administration. The Observatory was made possible by the generous financial support of the W. M. Keck Foundation. This research has made use of the Keck Observatory Archive (KOA), which is operated by the W. M. Keck Observatory and the NASA Exoplanet Science Institute (NExScI), under contract with the National Aeronautics and Space Administration. The research at Tennessee State University was supported in part by NASA, NSF, Tennessee State University, and the state of Tennessee through its Centers of Excellence program. Our work was also supported in part by NASA grants to UCLA.

\section{REFERENCES}

Anthony-Twarog, B. J., Deliyannis, C. P., Twarog, B. A., Croxall, K. V., \& Cummings, J. D. 2009, AJ, 138, 1191

Balachandran, S. 1995, ApJ, 446, 203

Barrado y Navascués, D., \& Stauffer, J. R. 1996, A\&A, 310, 879

Barrado y Navascués, D., Stauffer, J. R., Hartmann, L., \& Balachandran, S. 1997, Mem. Soc. Astron. Ital., 68, 939

Batten, A. H., Fletcher, J. M., \& MacCarthy, D. G. 1989, Dom. Astrophys. Obs., 17,1

Baumann, P., Ramírez, I., Meléndez, J., Asplund, M., \& Lind, K. 2010, A\&A, 519, A87

Boesgaard, A. M., \& Tripicco, M. J. 1986, ApJ, 302, L49

Bouvier, J. 2008, A\&A, 489, L53

Castelli, F., Gratton, R. G., \& Kurucz, R. L. 1997, A\&A, 318, 841

Chabrier, G., Baraffe, I., Allard, F., \& Hauschildt, P. 2000, ApJ, 542, 464

Cochran, W. D., Hatzes, A. P., Butler, R. P., \& Marcy, G. W. 1997, ApJ, 483, 457

Daniel, S. A., Latham, D. W., Mathieu, R. D., \& Twarog, B. A. 1994, PASP, 106,281

Demarque, P., Green, E. M., \& Guenther, D. B. 1992, AJ, 103, 151

Eaton, J. A., \& Williamson, M. H. 2004, Proc. SPIE, 5496, 710

Eaton, J. A., \& Williamson, M. H. 2007, PASP, 119, 886

Fitzpatrick, M. J. 1993, in ASP Conf. Ser. 52, Astronomical Data Analysis Software and Systems II, ed. R. J. Hanisch, R. V. J. Brissenden, \& J. Barnes (San Francisco, CA: ASP), 472

Gonzalez, G., Carlson, M. K., \& Tobin, R. W. 2010, MNRAS, 403, 1368

Gray, D. F. 1992, The Observation and Analysis of Stellar Photospheres (Cambridge: Cambridge Univ. Press)

Grevesse, N., \& Sauval, A. J. 1998, Space Sci. Rev., 85, 161 
Griffin, R. 1979, A Photometric Atlas of the Spectrum of Procyon (Cambridge: Cambridge Philosophical Society)

Harper, W. E. 1932, Publ. Dom. Astrophys. Obs., 7, 1

Israelian, G., Delgado Mena, E., Santos, N. C., et al. 2009, Nature, 462, 189

Jones, B. F., Fischer, D., \& Soderblom, D. R. 1999, AJ, 117, 330

Luck, R. E., \& Heiter, U. 2006, AJ, 131, 3069

Mashonkina, L., Gehren, T., Shi, J.-R., Korn, A. J., \& Grupp, F. 2011, A\&A, 528, A87

McLean, I. S., \& Sprayberry, D. 2003, Proc. SPIE, 4841, 1

Nidever, D. L., Marcy, G. W., Butler, R. P., Fischer, D. A., \& Vogt, S. S. 2002, ApJS, 141, 503

Nordström, B., Latham, D. W., Morse, J. A., et al. 1994, A\&A, 287, 338

Pasquini, L., Randich, S., \& Pallavicini, R. 1997, A\&A, 325, 535

Paulson, D. B., Sneden, C., \& Cochran, W. D. 2003, AJ, 125, 3185

Perryman, M. A. C., Brown, A. G. A., Lebreton, Y., et al. 1998, A\&A, 331, 81

Randich, S. 2009, in IAU Symp. 258, The Ages of Stars, ed. E. E. Mamajek, D. R. Soderblom, \& R. F. G. Wyse (Cambridge: Cambridge Univ. Press), 133

Randich, S. 2010, in IAU Symp. 268, Light Elements in the Universe, ed. C. Charbonnel, M. Tosi, F. Primas, \& C. Chiappini (Cambridge: Cambridge Univ. Press), 275

Reddy, B. E., Lambert, D. L., Laws, C., Gonzalez, G., \& Covey, K. 2002, MNRAS, 335, 1005

Scarfe, C. D., Batten, A. H., \& Fletcher, J. M. 1990, Publ. Dom. Astrophys. Obs., 18,21
Schuler, S. C., Cunha, K., Smith, V. V., et al. 2011, ApJ, 737, L32

Sestito, P., \& Randich, S. 2005, A\&A, 442, 615

Sestito, P., Randich, S., \& Pallavicini, R. 2004, A\&A, 426, 809

Sneden, C. 1973, ApJ, 184, 839

Song, I., Zuckerman, B., Weinberger, A., \& Becklin, E. E. 2005, Nature, 436, 363

Steffen, M. 1985, A\&AS, 59, 403

Stencel, R. E., \& Backman, D. E. 1991, ApJS, 75, 905

Thevenin, F. 1989, A\&AS, 77, 137

Thevenin, F. 1990, A\&AS, 82, 179

Thorburn, J. A., Hobbs, L. M., Deliyannis, C. P., \& Pinsonneault, M. H. 1993, ApJ, 415, 150

Tokovinin, A., Thomas, S., Sterzik, M., \& Udry, S. 2006, A\&A, 450, 681

Torres, G., Andersen, J., \& Giménez, A. 2010, A\&AR, 18, 67

van Leeuwen, F. 2007, Hipparcos, The New Reduction of the Raw Data (Dordrecht: Springer)

Vogt, S. S. 1987, PASP, 99, 1214

Weinberger, A. 2008, ApJ, 679, L41

Weinberger, A. J., Becklin, E. E., Song, I., \& Zuckerman, B. 2011, ApJ, 726, 72

Whitelock, P. A., Menzies, J. W., Catchpole, R. M., et al. 1991, MNRAS, 250, 638

Yelda, S., Lu, J. R., Ghez, A. M., et al. 2010, ApJ, 725, 331

Zuckerman, B., Fekel, F. C., Williamson, M. H., Henry, G. W., \& Muno, M. P. 2008, ApJ, 688, 1345 